\title{
Gut Microbiota and Metabolome Alterations Associated with Chinese Monks' Practice
}

\section{Tingting Qiao}

Department of Nuclear Medicine, Shanghai Tenth People's Hospital, Tongji University School of Medicine, Shanghai, China

\section{Ganghua Lu}

Department of Nuclear Medicine, Shanghai Tenth People's Hospital, Tongji University School of Medicine, Shanghai, China

\section{Zhongwei Lv}

Department of Nuclear Medicine, Shanghai Tenth People's Hospital, Tongji University School of Medicine, Shanghai, China

\section{Dan Li ( $\sim$ plumredlinda@163.com)}

Department of Nuclear Medicine, Shanghai Tenth People's Hospital, Tongji University School of Medicine, Shanghai, China

\section{Chengyou Jia}

Department of Nuclear Medicine, Shanghai Tenth People's Hospital, Tongji University School of Medicine, Shanghai, China

\section{Yingchun Song}

Department of Nuclear Medicine, Shanghai Tenth People's Hospital, Tongji University School of Medicine, Shanghai, China

\section{Wen Jiang}

Department of Nuclear Medicine, Shanghai Tenth People's Hospital, Tongji University School of Medicine, Shanghai, China

\section{Xiaqing Yu}

Department of Nuclear Medicine, Shanghai Tenth People's Hospital, Tongji University School of Medicine, Shanghai, China

\section{Dingwei Gao}

Department of Nuclear Medicine, Shanghai Tenth People's Hospital, Tongji University School of Medicine, Shanghai, China

Junyu Tong

Department of Nuclear Medicine, Shanghai Tenth People's Hospital, Tongji University School of Medicine, Shanghai, China

\section{Ru Wang}

Department of Nuclear Medicine, Shanghai Tenth People's Hospital, Tongji University School of Medicine, Shanghai, China

\section{Research Article}

Keywords: Chinese monks, Practice, Vegetarianism, Meditation, Gut microbiota, Fecal metabolites

Posted Date: January 10th, 2022

DOI: https://doi.org/10.21203/rs.3.rs-1198747/v1

License: (a) This work is licensed under a Creative Commons Attribution 4.0 International License. Read Full License 


\section{Abstract}

\section{Background}

The practices of monks mainly include long-term vegetarianism and meditation, which are likely to fundamentally influence the gut microbiota and fecal metabolites. We aim to study the relationship between the practices of Chinese monks and gut microbiotas and metabolites.

\section{Methods}

Twenty-four monks and forty-eight omnivorous controls (never meditated) were included. The microbiotas of all samples were profiled by $16 \mathrm{~S}$ rRNA gene sequencing, and the metabolomes were examined by nontargeted LC-MS metabolomics. Twenty-four monks were divided into the $\mathrm{H}$ group and the $\mathrm{L}$ group according to the median time of practice, and microbiota and metabolite analyses were carried out in the two groups.

Results

Microbial communities and metabolites were decreased in monks. Bacteroidetes was increased in monks, while the Firmicutes, Actinobacteria, and Firmicutes/Bacteroidetes ratios were decreased. At the genus level, Faecalibacterium, Lachnospira, Roseburia, norank_f_Lachnospiraceae, etc. were higher in monks, while Blautia, Eubacterium_hallii_group, Bifidobacteria, etc. were lower (all $p<0.05$ ). Most identical KEGG categories in both Tax4Fun and PICRUSt2 were related to metabolism (6/8, 75.0\%). Most higher abundance genera were positively correlated with higher abundance metabolites in monks, indicating that intestinal flora significantly affects intestinal metabolic function. Lipids and lipid-like molecules were the major differential metabolites (VIP $>2, p<0.05)$ in the two groups. L-dopa plays an important role in many metabolic pathways in monks. Prevotella_9 was enriched in the L group, while norank_f_Lachnospiraceae was enriched in the $\mathrm{H}$ group. DG (16:0/18:0/0:0) was highly expressed in the $\mathrm{H}$ group and participated in sixteen KEGG functional pathways as well as many immune-related KEGG enrichment pathways.

\section{Conclusion}

The monks' lifestyle practices of vegetarianism and meditation have the potential to modulate human metabolism and function by affecting the gut microbial composition and metabolites. The appropriate practice of monks makes the intestine younger and increases immunity, but long-term practice may cause adverse physical and mental events.

\section{Introduction}

Gut microbes have evolved with humans since the emergence of humans and have developed symbiotic relationships to ensure the survival of microbes and hosts[1]. Many studies have confirmed that the intestinal flora is closely related to the regulation of host immunity and inflammation[2,3]. Therefore, doctors treat a variety of diseases and improve human immunity by regulating intestinal microorganisms and intestinal metabolites, such as fecal bacteria transplantation $[4,5]$. Interestingly, there are many factors affecting the intestinal flora, among which dietary nutrition and meditation are regarded as important driving factors[6-10].

In recent years, many types of studies have shown that vegetarianism and meditation are beneficial for human physical and mental health. Different dietary habits affect the composition of intestinal flora and then influence physiological, metabolic, nutritional, and immune functions[7-11]. Vegetarianism effectively prevents metabolic diseases such as cardiovascular disease[9], diabetes[11], and obesity[8], as well as some cancers[12, 13]. Meditation by reducing stress and improving mental health has been widely studied for the treatment of various psychological, cardiovascular and digestive diseases, as well as relieving chronic pain[14, 15]. Additionally, meditation regulates overall health through the neuroendocrine-immune network[16-20], thus affecting the characteristics of the intestinal flora. 
As a special group, the practices of monks mainly includes the long-term vegetarian diet and focusing on meditation. Therefore, feces of monks are excellent data to study the synthetic effects of vegetarianism and meditation on intestinal changes. We aimed to investigate the effects of monks' long-term meditation and vegetarianism on the regulation of intestinal flora and metabolites.

\section{Results}

\section{The altered microbiota composition of monks compared with healthy omnivorous subjects}

We summarized the demographic information, including age, BW, BMI, and the practice lasting time of samples in the monk and control groups, as shown in Table 1. Eleven phyla, 221 genera, and 842 OTUs were obtained from 24 monk and 48 control stool samples. We confirmed that gut community structures significantly differed between the two groups in beta diversity $(p=0.001$, Figure $1 \mathrm{~A})$, and the monk group had a clearer structural separation of microbial communities by PCoA analysis (Figure 1B).

At the phylum level, the composition analysis of the intestinal microbial communities showed that both groups were dominated by four phyla; among them, the abundance of Bacteroides increased in monks, while the Firmicutes and Actinobacteria were increased in controls (Figure 1C, D, E). Interestingly, a significant difference in the Firmicutes/Bacteroidetes (F/B) ratio was observed between the two groups $(p<0.05$, Figure $1 \mathrm{~F})$. Furthermore, the type analysis according to the distribution of dominant organisms on JSD distance demonstrated that the samples were clustered into seven types (Figure 2A-B). Specifically, monks were clustered into types 1-3, while controls were clustered into types 17, suggesting that monks have a lower microbiota diversity of phyla. There were a greater number of type 1-2 cases in the monks, mainly including Firmicutes and Bacteroidetes (Figure 2B). Of note, Bacteroidetes accounted for the largest proportion in types 1-2 (Figure $2 \mathrm{C}$ ), which was consistent with the above results.

At the genus level, the Wilcoxon rank-sum test (mean abundance level of monk group $>1 \%, p<0.05$, Figure 2D) indicated 13 differentially expressed genera between the two groups. LEfSe analysis (LDA $>3.0, p<0.05$, Figure 2E) found that 33 genera could significantly distinguish the two groups. Specifically, the gut microbial composition characteristics in the two groups are shown in Figure 2F. Among 33 differential genera, we conducted random forest analysis to assess the value of these genera and selected the top nine important genera to serve as diagnostic biomarkers. The AUC of the ROC curve was 0.7795 [95\% confidence interval (CI): 0.6686-0.8904], Figure 3A), suggesting that these 9 genera could be employed as combined predictors. For the 9 genera, Eubacterium_eligens_group, Lachnospira, Roseburia, and norank_f_Lachnospiraceae were increased in monks, while Blautia, Dorea, Eubacterium_hallii_group, and Streptococcus were overexpressed in controls.

We analyzed the Spearman correlation between 33 differential genera and monks' general characteristics (Figure 3B). It is worth mentioning that the flora related to the monk's age and the practice duration was different. The results demonstrated that monk age was negatively correlated with Klebsiella (Rho $=-0.461, p=0.023)$. However, practice duration was positively correlated with norank_f_Lachnospiraceae (Rho $=0.542, p=0.006)$ but negatively correlated with Eubacterium_hallii_group $($ Rho $=-0.477, p=0.018)$. Therefore, the influence of monk practice duration on intestinal flora was different from that of age. Monk practice duration is an independent factor that influences intestinal flora.

Tax4Fun and PICRUSt2 were used to reveal the functional and metabolic-related changes in the gut microbiota in monks. We found that 308 KEGG categories were obtained using PICRUSt2, while 268 KEGG categories were obtained using Tax4Fun, indicating that the functional prediction of Tax4Fun was relatively conservative. The LEfSe analysis ( $\operatorname{LAD}>2.5, p<0.05$, Figure 3C-D) showed that 35 categories of Tax4Fun and 20 categories of PICRUSt2 were significantly different between the groups. For the eight identified common categories in both Tax4Fun and PICRUSt2 (Table 2 and Figure 3E), the majority were related to metabolism $(6 / 8,75.0 \%)$, including carbohydrate metabolism, energy metabolism, and glycan biosynthesis and metabolism, while the minority were related to environmental information processing $(2 / 8,25.0 \%)$, such as membrane 
transport. Notably, at KEGG pathway level 3, citrate cycle (TCA cycle), carbon fixation pathways in prokaryotes, other glycande gradation, and lipopolysaccharide biosynthesis were overrepresented in monks, while ABC transporters and phosphotransferase system (PTS) were overrepresented in controls. Spearman correlation was analyzed between the eight common KEGG categories and 33 differential genera (Figure 3F). The majority of the genera with the highest abundance in monks were positively correlated with the functional pathway enriched in the monk group, indicating that these ascendent genera influence the alteration of gut metabolism function.

\section{Analysis of metabolic spectrum and differences between the monks and the controls}

The nontargeted LC-MS metabolomics results demonstrated that a total of 34 differential metabolites $(\mathrm{VIP}>2, p<0.05)$ were successfully identified. A majority of differential metabolites were lipids and lipid-like molecules according to the HMDB database, as shown in Table S1. We used a PCA model with good performance to identify the distribution characteristics of metabolites between the groups (Figure 4A). VIP analysis was performed to visually display the importance of differential metabolites in the groups and the trend of expression (Figure 4B). The Kruskal test (Figure 4C) showed that 26 metabolites were significantly increased in monks, such as L-dopa and berberine, while only eight metabolites were significantly decreased, such as indoleacrylic acid, valyl-valine, and cadaverine.

The correlation analysis between the differential metabolites and general characteristics of monks

(Figure 4D) found that valyl-hydroxyproline, putreanine, lotaustralin, and N-docosanoyl taurine levels inversely correlated with practice duration, while $\mathrm{N}$-arachidonoyl tyrosine levels positively correlated with practice duration.

KEGG functional annotation analysis indicated that a majority of the KEGG pathways at level $2(6 / 12,50 \%)$ belong to the metabolism pathway (Figure 5A and Table S2). Surprisingly, L-Dopa was involved in seven of the twelve KEGG metabolic pathways. KEGG pathway enrichment analysis found that differential metabolites were enriched in thirteen metabolic pathways ( $p<0.05$, Figure 5B and Table S3), including biosynthesis of plant secondary metabolites, lysine degradation, tryptophan metabolism, cocaine addiction, amphetamine addiction, alcoholism, dopaminergic synapse, Parkinson disease, AMPK signaling pathway, and betalain biosynthesis. Interestingly, L-dopa was still involved in eleven of the thirteen enriched metabolic pathways. These results provide a valuable molecular basis for revealing the changes in intestinal function affected by monk practice.

Spearman correlation analysis examining 33 differential genera and 34 differential metabolites (Figure 5C) showed that most of the genera with higher abundance in monks were positively correlated with the metabolites with increased abundance in monks, indicating that most metabolites were influenced by the dominant intestinal microflora. We obtained some interesting correlations between flora and metabolites; for example, Eubacterium_eligens_group showed strong positive correlations with kynurenic acid (Rho $=0.6571, p<0.001)$, L-Dopa (Rho $=0.5756, p<0.001)$, etc. Lachnospira showed strong positive correlations with valyl-hydroxyproline (Rho $=0.6048, p<0.001$ ), 6-keto PGE1 (Rho $=0.5551, p<0.001$ ), and kynurenic acid $(\mathrm{Rho}=0.5512, p<0.001)$. Ruminococcaceae_UCG-003 showed strong positive correlations with kynurenic acid (Rho = $0.5652, p<0.001)$, L-Dopa (Rho $=0.5488, p<0.001)$, and PE $(14: 0 / 0: 0)(\mathrm{Rho}=0.5217, p<0.001)$. Additionally, correlation analysis between differential genera and the twelve lipids and lipid-like metabolites (Figure 5D) showed a strong correlation between the lipid compounds and the enriched genera in monks.

\section{The monks' practice time significantly affected the composition of the microbiota and gut metabolome}

Based on the above analysis, we found that the practice lasting time of vegetarianism and meditation was of great significance to the alterations of intestinal flora and metabolites in monks. To further explore the impact of the practice duration on intestinal changes, the monks were divided into $\mathrm{H}$ groups and $\mathrm{L}$ group using the median 7.5 of the practice duration (Table 3). PCoA analysis showed that the microbial composition of the $\mathrm{H}$ group was significantly different from that of the $L$ group (Figure 6A). The Wilcoxon rank-sum test $(p<0.05)$ and LEfSe analysis (LDA $>2.0, p<0.05)$ found that norank_f_Lachnospiraceae and norank_c_Cyanobacteria were overrepresented in the H group, while Prevotella_9, 
Bifidobacterium, unclassified_f_Peptostreptococcaceae, Gordonibacter and Enterococcus were overrepresented in the L group (Figure 6B-C).

To reveal the effects of practice duration on the functional and metabolic-related changes of gut microbiota, PICRUSt2 (321 KEGG categories) and Tax4Fun (268 KEGG categories) identified a total of 227 common KEGG pathways. Based on the LEfSe analysis ( $L A D>2.0, P<0.05$, Figure 6D-E and Table 4-5), Tax4Fun or PICRUSt2 screened 12 KEGG pathway level 3 pathways with significant differences between the two groups. Notably, valine leucinean disoleucine degradation (ko00280) and peptidoglycan biosynthesis (ko00550) were identical in Tax4Fun and PICRUSt2. The former belonged to amino acid metabolism and was highly expressed in the $\mathrm{H}$ group, and the latter belonged to glycan biosynthesis and metabolism and was highly expressed in the L group. Additionally, dopaminergic synapses (ko04728) of the nervous system were enriched in the L group.

Spearman correlation was utilized to analyze the potential connections between the seven differential genera and twelve KEGG pathway level 3 of Tax4Fun or PICRUSt2 (Figure 6F-G). In Tax4Fun, Prevotella_9 was positively correlated with the highly expressed KEGG pathways in the $L$ group but negatively correlated with the highly expressed KEGG pathways in the $H$ group. Interestingly, Prevotella_9 was negatively correlated with all KEGG pathways in PICRUSt2.

A total of 23 differential metabolites (VIP $>2, p<0.05$ ) were successfully identified in the $\mathrm{H}$ and $\mathrm{L}$ groups, which were classified into organoheterocyclic compounds, organic oxygen compounds, organic acids and derivatives, lipids and lipid-like molecules, and benzenoids according to the HMDB database, as shown in Table S4. The PCA model indicated that the two groups were different from each other (Figure 7A). The Kruskal-Wallis test (Figure 7B) showed that fifteen metabolites were upregulated in the H group, such as DG (16:0/18:0/0:0), 5-Hydroxyflavone, 2-Linoleoyl Glycerol, and Glycocholic Acid, while Incensole oxide, Valyl-Hydroxyproline, Ginkgoic acid, and Acetyl-L-tyrosine, were significantly reduced.

For KEGG functional pathways, differential metabolites were involved in the five primary KEGG pathways, containing 19 secondary pathways (Figure 7C). Notably, DG (16:0/18:0/0:0) participated in eight organic systems pathways, six human diseases pathways, one environmental information processing pathway and one cellular process, as shown in Table S5. Based on KEGG enrichment pathway analysis, differential metabolites were enriched in 67 metabolic pathways $(p<0.05)$, and the top 20 enrichment pathways in ER values are shown in Figure 7D. Many enrichment pathways were related to immunity, such as Th1 and Th2 cell differentiation, Th17 cell differentiation, B cell receptor signaling pathway, natural killer cell-mediated cytotoxicity, and T cell receptor signaling pathway. Surprisingly, DG (16:0/18:0/0:0) was also involved in these immune-related pathways.

\section{Discussion}

We aimed to study the regulation of Chinese monks' gut microbiota and metabolome alterations from the practices of meditation and vegetarianism, displayed an outline of the complex alterations interrelated with Chinese monks in the gut microbiota as well as the intestinal metabolites in Chinese monks, and explored the correlation between the gut changes and the practice duration of monks. We obtained many interesting results, which display the characterizations of microbial community composition and metabolites in human feces and provide a brand and new idea to understand the molecular phenotypic differences between Chinese monks with meditation and vegetarian lifestyles and controls with omnivorous and nonmeditation lifestyles.

We found that Firmicutes were lower and Bacteroides were higher in monks, and the Firmicutes/Bacteroidetes ratio was decreased in monks. Previous studies have reported that Firmicutes increased in obese people, while Bacteroides increased in thin people[21, 22]. Some studies have suggested an increased F/B ratio as one marker of obesity[23]. Moreover, the F/B ratio was lower in young people[24]. Monks are long-term vegetarians and rarely eat fatty food. Additionally, monks meditate many times per day, which activates thinking and relaxes the body; thus, monks have less pressure than peers without practice 
lifestyles, making bodies and minds younger. We believe that the above factors cause a decline in the F/B ratio in monks. However, monks never eat meat in their diets, resulting in the lack of sufficient protein and some essential trace elements, which lead to a decline in intestinal microbial diversity.

Previous research found that Roseburia, Eubacterium_eligens_group, norank_f_Lachnospiraceae, and Lachnospiraceae_NK4A136_group were dominant bacterial genera in the vegan meditation group[6], which was consistent with our research showing that these genera were significantly increased in monks. Roseburia is a common butyric acidproducing bacterium and one key bacterium degrading dietary fiber xylan in the human intestine[25,26]. Studies have shown that colonization of butyric acid-producing intestinal Roseburia bacteria in mice can reduce inflammatory markers and improve atherosclerosis under the influence of a diet rich in plant polysaccharides[25], indicating that Roseburia has a beneficial effect on the cardiovascular system. Faecobacteria is one of the most important butyric-producing bacteria in the human colon and is considered a bioindicator of human health[27]. Inflammatory process development promoted by Faecobacteria can be reduced. Roseburia and Faecalibacterium significantly increased in monks, showing that long-term vegan meditation promotes the growth of butyric acid-producing bacteria and plays a positive role in health. Lachnospira promotes the efficacy of antihistamines in chronic spontaneous urticaria (CSU), which is a signature to predict the efficacy of antihistamines in CSU patients[28]. Lachnospira increased in monks, suggesting that CSU patients may improve the efficacy of antihistamines by the practices of vegetarians and meditation. It has been reported that the Eubacterium_hallii_group is the dominant bacteria in chronic pancreatitis[29], and pancreatitis is closely related to overeating and hyperlipidemia. Eubacterium_hallii_group significantly decreased in monks, which is consistent with the healthy practices of long-term vegetarian monks. However, long-term vegetarian diets inevitably caused a decrease in some beneficial bacteria, such as Bifidobacteria and Blautia. Bifidobacterium is a beneficial bacteria physiologically with various functions, such as nutrition[30], enhancing immunity[31], improving gastrointestinal function[32], preventing cancer[33], and antiaging. Blautia can prevent intestinal inflammation, promote the production of short-chain fatty acids and maintain intestinal steady-state activity, which has potential probiotic properties[35]. Thus, the reduction of Bifidobacteria and Blautia led to a negative impact on intestinal balance in monks.

Citrate cycle, carbon fixation pathways in prokaryotes, other glycande gradation, and lipopolysaccharide biosynthesis were enriched in monks, showing that the energy metabolism of monks was higher than that of controls. This suggests that vegetarianism and meditation affect changes in the intestinal flora and metabolites, thus affecting the absorption of intestinal nutrients and finally causing changes in monk body metabolism. Of note, ABC transporters and PTS were inhibited in monks, which is beneficial to health. Some studies have proven that PTS plays a role in antimicrobial resistance. For example, glycocins are antimicrobial glycopeptides that are regarded as novel targets, and PTS affects the antibacterial activity of glycocins[36]. Another study showed that cajanin stilbene acid killed Enterococcus by inhibiting the PTS of Enterococcus[37], thus hindering the normal growth and metabolic functions of bacteria. As a drug transporter in a variety of tumors, $A B C$ transporters are considered to be the major participant in tumor chemoresistance and play a key role in the development of tumors[38-40]. Therefore, monks may be less likely to develop drug resistance than controls.

We identified twelve lipids and lipid-like molecules of differential metabolites, which were increased in monks. Previous research has found that lipids and lipid-like molecules were significantly increased in type 2

diabetes patients[41]. We believe that monks have lipid dysregulation due to a long-term carbohydrate-based vegetarian diet, which may require early intervention and treatment. It should be noted that L-dopa, a neurotransmitter involved in happiness and the most commonly used drug to treat Parkinson's disease[42-44], was significantly increased in monks. L-Dopa plays an important role in multiple KEGG pathways, which may be related to the long-term meditation of monks' practice. Meditation can reduce stress and anxiety and enhance memory and concentration. Thus, we suggest that high-pressure people be happy by increasing L-dopa via the lifestyle of monks' practice. Additionally, Parkinson's disease patients might improve their condition via the monks' practice lifestyle. Kynurenic acid, as the major metabolite of tryptophan oxidative metabolism, regulates neuronal excitability and plasticity, brain development, and behavior and plays a protective role in Huntington's disease $[45,46]$. Therefore, we believe that the long-term meditation of monks promotes the brain to think, protects the function of the brain, and prevents neurodegenerative diseases. Many studies have reported that solasodine[47], peimine[48], 
berberine[49], ginkgoic acid[50], and 6-pentadecyl salicylic acid[51] have antimetastatic effects and inhibit tumor cell proliferation in various cancers. Therefore, the practices of meditation and vegetarianism may help monks prevent cancer.

A significant correlation between dominant microbiotas and enriched metabolic pathways was observed in monks, indicating that gut metabolic phenotype alterations were associated with microbiota changes. Lysine degradation is an important link in human central metabolism and is associated with human cancer[52, 53]. The AMPK signaling pathway is related to cell metabolism and tumor autophagy $[54,55]$. These results coincided with the functions of the identified microbiota and metabolites in monks; that is, meditation and vegetarianism of monks have an impact on metabolism and cancers. The pathways of cocaine addiction[56], amphetamine addiction, alcoholism, dopaminergic synapses, and Parkinson's disease were related to mental states or mental illnesses, indicating that meditation of monks plays a part in the "gut-brain" axis, but further experimental verification is still needed to confirm this hypothesis.

The practice duration among monks has a significant impact on the composition of intestinal flora. Interestingly, we found that norank_f__ Lachnospiraceae was significantly increased not only in the monk group but also in the $\mathrm{H}$ group, indicating that norank_f__ Lachnospiraceae is an important genus that affects intestinal changes through monks' practice lifestyle. It has been reported that norank_f__ Lachnospiraceae is one of the dominant bacteria in autism spectrum disorder[57], so we speculated that norank_f__ Lachnospiraceae may be one of the significant bacteria on the "gut-brain" axis. In addition, norank_f__ Lachnospiraceae could alleviate body weight gain, visceral fat accumulation, dyslipidemia, and impaired glucose tolerance induced by a high-fat diet[58]. This conformed to the general point that the long-term practice of monks prevents the body from harming the fatty diet. Moreover, Prevotella_9 and Bifidobacterium were reduced in the long-term practice group, showing that Provotella_9 and Bifidobacterium lose their initial advantage in the monk gut with the extension of practice time. Many studies have reported that Prevotella is a common human intestinal bacterium that is related to vegetarian diets and is a dominant flora in carbohydrate diets[59,60].

The majority of differential metabolites of the $\mathrm{H}$ and $\mathrm{L}$ groups were lipids and lipid-like molecules, indicating that the longer monks practiced, the higher the risk of lipid metabolism disorder. Notably, DG (16:0/18:0/0:0) was involved in the metabolic pathway of long-term depression enrichment, which was highly expressed in the $\mathrm{H}$ group. Therefore, we further speculated that the appropriate practice of monks could improve the sense of happiness, reduce life pressure, and improve the mental state, but long-term practice may lead to depression. Additionally, DG (16:0/18:0/0:0) is involved in many immune-related pathways, such as natural killer cell-mediated cytotoxicity, Th17 cell differentiation, the B cell receptor signaling pathway, the T cell receptor signaling pathway, and Th1 and Th2 cell differentiation. Thus, we inferred that the long-term practice of monks might stimulate some immune responses in the intestines to protect or harm the body, which is related to long-term vegetarianism or meditation. DG (16:0/18:0/0:0) participated in the circular system, digestive system, endocrine system, environmental adaptation, excess system, immune system, nervous system, and sensory system in the KEGG functional pathway, suggesting that the organic systems of the whole body would be affected with prolonged practice. The above results reflected that DG (16:0/18:0/0:0) plays an important role in the intestinal metabolism of monks, but the specific molecular mechanism is unknown, which is the focus of further research.

In summary, our data highlighted that gut microbiota and metabolite alterations are associated with the practices of monks, suggesting that vegetarianism and meditation have an important impact on intestinal stability. We hold the view that proper practice of monks is conducive to physical and mental health, but long-term practices might lead to physical and mental adverse events.

\section{Materials And Methods}

\section{Study design}

To investigate the gut microbiota and metabolites in monks, we recruited a total of 24 monks with the practices of long-term vegetarianism and meditation from Zhengfa Temple of Wuxi City, Jiangsu Province. Other key information, including age, 
body weight (BW), body mass index (BMI), and the duration of monk use, was obtained when collecting monk fecal samples.

To avoid the influence of gender on the results, we selected 48 healthy male omnivores who did not mediate as the control group. This protocol in this study was approved by the ethics committee of Shanghai Tenth People's Hospital (ethics No.: shsy - IEC - ki - 4.0/16-18/01). All subjects signed informed consent forms and were informed of the nature of the study prior to the collection of stool samples.

\section{DNA extraction, PCR amplification, and MiSeq sequencing}

Fecal samples of participants were collected using sterile tubes and stored in liquid nitrogen at $-80{ }^{\circ} \mathrm{C}$ until they were used for the study of extracting microbial DNA and metabolites. 16S rRNA gene sequencing was used to analyze the fecal microbiota. The E.Z.N.A. ® Soil DNA Kit (Omega Bio-Tek, Norcross, GA) was used to extract bacterial DNA from fecal samples. A Nanodrop2000 was used to determine the concentration of bacterial DNA. The extracted DNA was stored at -20 ${ }^{\circ} \mathrm{C}$ to prevent degradation. The V3-V4 variable region of the $16 \mathrm{~S}$ rRNA gene was amplified by PCR with specific primers $338 \mathrm{~F}$ (5'-ACTCCTACGGGAGGCAGCAG-3') and 806R (GGACTACHVGGGTWTCTAAT-3'). Each sample was repeated three times to ensure the reliability of the subsequent microbiological analysis. The amplified products were purified by an AxyPrep DNA Extraction Kit (AxyPrep Biosciences, USA) and quantified by quantitative TM st (Promega, USA). Then, the purified amplicons were combined and sequenced on the Illumina MiSeq PE300 platform (Illumina San Diego, USA).

\section{Fecal metabolite extraction}

The $400-\mu \mathrm{L}$ methanol:water $(4: 1, \mathrm{~V} / \mathrm{V})$ solution was thoroughly mixed with a $50-\mathrm{mg}$ stool sample and then crushed at $50 \mathrm{~Hz}$ for 6 min using a WONBio-96C high-throughput tissue crusher (Shanghai Wanbo Biotechnology Co., Ltd.). The mixture was then rotated for 30 seconds and treated with ultrasound for 30 minutes at $40 \mathrm{kHz}$ and $5^{\circ} \mathrm{C}$. The mixture was then incubated at $-20{ }^{\circ} \mathrm{C}$ for 30 minutes to precipitate the protein. After centrifugation at $4{ }^{\circ} \mathrm{C}$ at $13,000 \mathrm{~g}$ for $15 \mathrm{~min}$, the supernatant was transferred to the sample flask, and positive and negative ions were analyzed by UPLC QTOF MS. Additionally, 10 $\mu \mathrm{L}$ supernatant was mixed from each sample as a quality control (QC) sample and injected periodically (every 8 samples) to monitor the stability of the analysis.

\section{Microbial analysis of 16S rRNA gene sequencing}

We used the Majorbio Cloud Platform (https://cloud.majorbio.com/) to analyze the 16S rRNA gene sequencing data. According to the overlap relationship, the paired end fragments obtained from Illumina MiSeq PE300 high-throughput sequencing were spliced into the target sequence for quality control screening. We eliminated the chimera and optimized the sequence by UCHIME software. The Ribosome Database Project (RDP) classifier (version 2.2, http://sourceforge.net/projects/rdp-classifier/) was in the Silva database (version 128, https://www.arbsilva.de/documentation/release-Use 128/) to classify and label the optimal sequence with a confidence threshold of $70 \%$. UPARSE software (version 7.1, http://drive5. COM/UPARSE/) was used to cluster operational taxa (OTUs) with a similarity of $97 \%$. To avoid pseudosequences, OTUs accounting for less than $0.005 \%$ of the total number of sequences were deleted.

\section{Data preprocessing of LC-MS/MS}

LC-MS raw data were imported into Progenesis QI 2.3 (Nonlinear Dynamics, Waters, USA) for peak detection and comparison. Then, a data matrix consisting of retention time (RT), mass charge ratio $(\mathrm{m} / \mathrm{z})$ value and peak intensity was generated. Qualitative and quantitative results for specific metabolites were obtained. It is important to note that metabolic datasets must be preprocessed prior to multivariate statistical analysis. The pretreatment methods were as follows:

1) data filtering included removing samples with missing values $>50 \%$; 2 ) missing values were simulated according to half of the minimum metabolite values, and the total peak was normalized; and 3) elimination of QC samples with a relative standard deviation (RSD) $>30 \%$. Finally, after logarithmic conversion, a preprocessed dataset is prepared for further analysis.

\section{Bioinformatic analysis and multivariate statistical analysis}

Page $8 / 22$ 
In the Majorbio Cloud Platform, beta diversity analyses were calculated using the score plot of principal coordinate analysis (PCOA) and analysis of similarities (ANOSIM) to estimate whether intergroup differences were significantly greater than intragroup differences. The Wilcoxon rank-sum test was used to compare the differences in gut microbiota between the groups. Linear discriminant analysis effect size (LEfSe) analysis with LDA score was used to determine the differential taxa or functional pathways between the groups[61]. The typing analysis by the Partitioning Around Medoids algorithm was used to cluster all samples into seven types. Random forest model analysis with default settings was performed in Majorbio. Based on the Kyoto Encyclopedia of Genes and Genomes (KEGG) database, Tax4Fun and PICRUST2 were also used to perform functional pathway analysis in Majorbio. Venn analysis was performed using the OECloud tools at https://cloud.oebiotech.cn. Spearman correlation analysis with species clusters was performed using the OmicStudio tools (https://www.omicstudio.cn/tool), and the results are presented as a heatmap. Additionally, the receiver operating characteristic (ROC) curve with the area under the curve (AUC) was constructed in OmicStudio.

SIMCA software (V16.0.2, Sartorius Stedim Data Analytics AB, Umea, Sweden) was used to analyze the nontargeted LC-MS metabolomics. Principal component analysis (PCA) was used to visualize the distribution characteristics of metabolites between the groups. We used the PCA model to generate the corresponding VIP values to reflect the contribution of the distribution between groups. The metabolites were considered to differ significantly between groups when VIP values $>2$ and $p<0.05$. Differential metabolites were mapped to their biochemical pathways based on the KEGG database using MetboAnalyst 5.0 (http://www.metaboanalyst.ca/).

\section{Declarations}

\section{§ Ethics approval and consent to participate}

This protocol in this study was approved by the ethics committee of Shanghai Tenth People's Hospital (ethics No.: shsy- IEC$\mathrm{ki}-4.0 / 16-18 / 01)$. All subjects signed informed consents and were informed of the nature of the study prior to the collection of stool samples.

\section{$\S$ Consent for publication}

Not applicable.

\section{$\S$ Availability of data and material}

Please contact author for data requests.

\section{$\S$ Competing interests}

The authors declare that they have no competing interests.

\section{§ Funding}

This research was funded by National Natural Science Foundation of China (Grant No. 82071964), Shanghai Shenkang Three-year Action Project (Grant No. SHDC2020CR2054B), Key discipline construction project of the three-year action plan of Shanghai public health system (Grant No. GWV-10.1-XK9), and Shanghai Natural Science Foundation(Grant No. 21ZR1449600). National Natural Science Foundation of China and Shanghai Shenkang Three-year Action Project offered the funding for testing samples. Key discipline construction project of the three-year action plan of Shanghai public health system and Shanghai Natural Science Foundation offered the funding for analysing the data.

\section{§ Authors' contributions}

QTT, LZW, and LD conceived the study and participated in research design and data interpretation; QTT, JCY, and LGH contributed significantly to analysis and manuscript preparation; QTT and LGH performed the data analyses, prepared figures 
and tables, and wrote the manuscript; LZW, LD, SYC, JW, YXQ, GDW, TJY, and WR helped perform the analysis with constructive discussions; LD helped revise the article, and LZW offered all the fundings.

\section{$\S$ Acknowledgements}

Not applicable.

\section{References}

[1] Ley RE, Peterson DA, Gordon JI. Ecological and evolutionary forces shaping microbial diversity in the human intestine. CELL. 2006 2006-02-24;124(4):837-48.

[2] Round JL, Mazmanian SK. The gut microbiota shapes intestinal immune responses during health and disease. NAT REV IMMUNOL. 2009 2009-05-01;9(5):313-23.

[3] Sethi V, Vitiello GA, Saxena D, Miller G, Dudeja V. The Role of the Microbiome in Immunologic Development and its Implication For Pancreatic Cancer Immunotherapy. GASTROENTEROLOGY. 2019 2019-05-01;156(7):2097-115.

[4] Battipaglia G, Malard F, Rubio MT, Ruggeri A, Mamez AC, Brissot E, et al. Fecal microbiota transplantation before or after allogeneic hematopoietic transplantation in patients with hematologic malignancies carrying multidrug-resistance bacteria. HAEMATOLOGICA. 2019 2019-08-01;104(8):1682-8.

[5] Paramsothy S, Nielsen S, Kamm MA, Deshpande NP, Faith JJ, Clemente JC, et al. Specific Bacteria and Metabolites Associated With Response to Fecal Microbiota Transplantation in Patients With Ulcerative Colitis. GASTROENTEROLOGY. 2019 2019-04-01;156(5):1440-54.

[6] Jia W, Zhen J, Liu A, Yuan J, Wu X, Zhao P, et al. Long-Term Vegan Meditation Improved Human Gut Microbiota. Evid Based Complement Alternat Med. 2020 2020-01-20;2020:9517897.

[7] Wu GD, Compher C, Chen EZ, Smith SA, Shah RD, Bittinger K, et al. Comparative metabolomics in vegans and omnivores reveal constraints on diet-dependent gut microbiota metabolite production. GUT. 2016 2016-01-01;65(1):63-72.

[8] Losasso C, Eckert EM, Mastrorilli E, Villiger J, Mancin M, Patuzzi I, et al. Assessing the Influence of Vegan, Vegetarian and Omnivore Oriented Westernized Dietary Styles on Human Gut Microbiota: A Cross Sectional Study. FRONT MICROBIOL. 2018 2018-01-20;9:317.

[9] Do RV, Fernandes R, Trindade EB. Vegetarian diets and gut microbiota: important shifts in markers of metabolism and cardiovascular disease. NUTR REV. 2016 2016-07-01;74(7):444-54.

[10] Matijasic BB, Obermajer T, Lipoglavsek L, Grabnar I, Avgustin G, Rogelj I. Association of dietary type with fecal microbiota in vegetarians and omnivores in Slovenia. EUR J NUTR. 2014 2014-06-01;53(4):1051-64.

[11] Kahleova H, Matoulek M, Malinska H, Oliyarnik O, Kazdova L, Neskudla T, et al. Vegetarian diet improves insulin resistance and oxidative stress markers more than conventional diet in subjects with Type 2 diabetes. Diabet Med. 2011 2011-05-01;28(5):549-59.

[12] Anyene IC, Ergas IJ, Kwan ML, Roh JM, Ambrosone CB, Kushi LH, et al. Plant-Based Dietary Patterns and Breast Cancer Recurrence and Survival in the Pathways Study. NUTRIENTS. 2021 2021-09-25;13(10).

[13] Ratjen I, Enderle J, Burmeister G, Koch M, Nothlings U, Hampe J, et al. Post-diagnostic reliance on plant-compared with animal-based foods and all-cause mortality in omnivorous long-term colorectal cancer survivors. AM J CLIN NUTR. 2021 2021-08-02;114(2):441-9. 
[14] Black DS, Slavich GM. Mindfulness meditation and the immune system: a systematic review of randomized controlled trials. Ann N Y Acad Sci. 2016 2016-06-01;1373(1):13-24.

[15] Carlson LE, Speca M, Faris P, Patel KD. One year pre-post intervention follow-up of psychological, immune, endocrine and blood pressure outcomes of mindfulness-based stress reduction (MBSR) in breast and prostate cancer outpatients. BRAIN BEHAV IMMUN. 2007 2007-11-01;21(8):1038-49.

[16] Jacobs TL, Epel ES, Lin J, Blackburn EH, Wolkowitz OM, Bridwell DA, et al. Intensive meditation training, immune cell telomerase activity, and psychological mediators. PSYCHONEUROENDOCRINO. 2011 2011-06-01;36(5):664-81.

[17] Holzel BK, Lazar SW, Gard T, Schuman-Olivier Z, Vago DR, Ott U. How Does Mindfulness Meditation Work? Proposing Mechanisms of Action From a Conceptual and Neural Perspective. PERSPECT PSYCHOL SCI. 2011 2011-11-01;6(6):537-59.

[18] Creswell JD, Taren AA, Lindsay EK, Greco CM, Gianaros PJ, Fairgrieve A, et al. Alterations in Resting-State Functional Connectivity Link Mindfulness Meditation With Reduced Interleukin-6: A Randomized Controlled Trial. Biol Psychiatry. 2016 2016-07-01;80(1):53-61.

[19] Househam AM, Peterson CT, Mills PJ, Chopra D. The Effects of Stress and Meditation on the Immune System, Human Microbiota, and Epigenetics. Adv Mind Body Med. 2017 2017-07-01;31(4):10-25.

[20] Pascoe MC, Thompson DR, Ski CF. Meditation and Endocrine Health and Wellbeing. Trends Endocrinol Metab. 2020 2020-07-01;31(7):469-77.

[21] Koliada A, Syzenko G, Moseiko V, Budovska L, Puchkov K, Perederiy V, et al. Association between body mass index and Firmicutes/Bacteroidetes ratio in an adult Ukrainian population. BMC MICROBIOL. 2017 2017-05-22;17(1):120.

[22] Stojanov S, Berlec A, Strukelj B. The Influence of Probiotics on the Firmicutes/Bacteroidetes Ratio in the Treatment of Obesity and Inflammatory Bowel disease. Microorganisms. 2020 2020-11-01;8(11).

[23] Magne F, Gotteland M, Gauthier L, Zazueta A, Pesoa S, Navarrete P, et al. The Firmicutes/Bacteroidetes Ratio: A Relevant Marker of Gut Dysbiosis in Obese Patients? NUTRIENTS. 2020 2020-05-19;12(5).

[24] Vaiserman A, Romanenko M, Piven L, Moseiko V, Lushchak O, Kryzhanovska N, et al. Differences in the gut Firmicutes to Bacteroidetes ratio across age groups in healthy Ukrainian population. BMC MICROBIOL. 2020 2020-0722;20(1):221.

[25] Kasahara K, Krautkramer KA, Org E, Romano KA, Kerby RL, Vivas El, et al. Interactions between Roseburia intestinalis and diet modulate atherogenesis in a murine model. NAT MICROBIOL. 2018 2018-12-01;3(12):1461-71.

[26] Leth ML, Ejby M, Workman C, Ewald DA, Pedersen SS, Sternberg C, et al. Differential bacterial capture and transport preferences facilitate co-growth on dietary xylan in the human gut. NAT MICROBIOL. 2018 2018-05-01;3(5):570-80.

[27] Ferreira-Halder CV, Faria A, Andrade SS. Action and function of Faecalibacterium prausnitzii in health and disease. Best Pract Res Clin Gastroenterol. 2017 2017-12-01;31(6):643-8.

[28] Liu R, Peng C, Jing D, Xiao Y, Zhu W, Zhao S, et al. Lachnospira is a signature of antihistamine efficacy in chronic spontaneous urticaria. EXP DERMATOL. 2021 2021-09-24.

[29] Zhou CH, Meng YT, Xu JJ, Fang X, Zhao JL, Zhou W, et al. Altered diversity and composition of gut microbiota in Chinese patients with chronic pancreatitis. PANCREATOLOGY. 2020 2020-01-01;20(1):16-24.

[30] Kelly SM, Munoz-Munoz J, van Sinderen D. Plant Glycan Metabolism by Bifidobacteria. FRONT MICROBIOL. 2021 2021-01-20;12:609418. 
[31] Wang F, Yin Q, Chen L, Davis MM. Bifidobacterium can mitigate intestinal immunopathology in the context of CTLA4 blockade. Proc Natl Acad Sci U S A. 2018 2018-01-02;115(1):157-61.

[32] Monteagudo-Mera A, Arthur JC, Jobin C, Keku T, Bruno-Barcena JM, Azcarate-Peril MA. High purity galactooligosaccharides enhance specific Bifidobacterium species and their metabolic activity in the mouse gut microbiome. BENEF MICROBES. 2016 2016-01-20;7(2):247-64.

[33] Faghfoori Z, Faghfoori MH, Saber A, Izadi A, Yari KA. Anticancer effects of bifidobacteria on colon cancer cell lines. CANCER CELL INT. 2021 2021-05-12;21(1):258.

[34] Kim H, Kim S, Park SJ, Park G, Shin H, Park MS, et al. Administration of Bifidobacterium bifidum BGN4 and Bifidobacterium longum BORI Improves Cognitive and Memory Function in the Mouse Model of Alzheimer's Disease. FRONT AGING NEUROSCI. 2021 2021-01-20;13:709091.

[35] Liu X, Mao B, Gu J, Wu J, Cui S, Wang G, et al. Blautia-a new functional genus with potential probiotic properties? Gut Microbes. 2021 2021-01-01;13(1):1-21.

[36] Biswas S, Wu C, van der Donk WA. The Antimicrobial Activity of the Glycocin Sublancin Is Dependent on an Active Phosphoenolpyruvate-Sugar Phosphotransferase System. ACS INFECT DIS. 2021 2021-08-13;7(8):2402-12.

[37] Tan S, Hua X, Xue Z, Ma J. Cajanin Stilbene Acid Inhibited Vancomycin-Resistant Enterococcus by Inhibiting Phosphotransferase System. FRONT PHARMACOL. 2020 2020-01-20;11:473.

[38] Strachowska M, Gronkowska K, Michlewska S, Robaszkiewicz A. CBP/p300 Bromodomain Inhibitor-I-CBP112 Declines Transcription of the Key ABC Transporters and Sensitizes Cancer Cells to Chemotherapy Drugs. Cancers (Basel). 2021 2021-09-14;13(18).

[39] Serra M, Hattinger CM, Pasello M, Casotti C, Fantoni L, Riganti C, et al. Impact of ABC Transporters in Osteosarcoma and Ewing's Sarcoma: Which Are Involved in Chemoresistance and Which Are Not? CELLS-BASEL. 2021 2021-09-17;10(9).

[40] Thurm C, Schraven B, Kahlfuss S. ABC Transporters in T Cell-Mediated Physiological and Pathological Immune Responses. INT J MOL SCl. 2021 2021-08-25;22(17).

[41] Hameed A, Mojsak P, Buczynska A, Suleria H, Kretowski A, Ciborowski M. Altered Metabolome of Lipids and Amino Acids Species: A Source of Early Signature Biomarkers of T2DM. J CLIN MED. 2020 2020-07-16;9(7).

[42] LeWitt PA. Levodopa therapy for Parkinson's disease: Pharmacokinetics and pharmacodynamics. Mov Disord. 2015 2015-01-01;30(1):64-72.

[43] Haaker J, Gaburro S, Sah A, Gartmann N, Lonsdorf TB, Meier K, et al. Single dose of L-dopa makes extinction memories context-independent and prevents the return of fear. Proc Natl Acad Sci U S A. 2013 2013-06-25;110(26):E242836.

[44] Rutledge RB, Skandali N, Dayan P, Dolan RJ. Dopaminergic Modulation of Decision Making and Subjective WellBeing. J NEUROSCI. 2015 2015-07-08;35(27):9811-22.

[45] Szalardy L, Zadori D, Toldi J, Fulop F, Klivenyi P, Vecsei L. Manipulating kynurenic acid levels in the brain - on the edge between neuroprotection and cognitive dysfunction. CURR TOP MED CHEM. 2012 2012-01-20;12(16):1797-806.

[46] Fukuwatari T. Possibility of Amino Acid Treatment to Prevent the Psychiatric Disorders via Modulation of the Production of Tryptophan Metabolite Kynurenic Acid. NUTRIENTS. 2020 2020-05-13;12(5). 
[47] Fan Y, Li Z, Wu L, Lin F, Shao J, Ma X, et al. Solasodine, Isolated from Solanum sisymbriifolium Fruits, Has a Potent Anti-Tumor Activity Against Pancreatic Cancer. Drug Des Devel Ther. 2021 2021-01-20;15:1509-19.

[48] Tan H, Zhang G, Yang X, Jing T, Shen D, Wang X. Peimine inhibits the growth and motility of prostate cancer cells and induces apoptosis by disruption of intracellular calcium homeostasis through $\mathrm{Ca}(2+) / \mathrm{CaMKII/JNK}$ pathway. J CELL BIOCHEM. 2020 2020-01-01;121(1):81-92.

[49] Chuang TC, Wu K, Lin YY, Kuo HP, Kao MC, Wang V, et al. Dual down-regulation of EGFR and ErbB2 by berberine contributes to suppression of migration and invasion of human ovarian cancer cells. ENVIRON TOXICOL. 2021 2021-0501;36(5):737-47.

[50] Liu D, Li Z, Yang Z, Ma J, Mai S. Ginkgoic acid impedes gastric cancer cell proliferation, migration and EMT through inhibiting the SUMOylation of IGF-1R. Chem Biol Interact. 2021 2021-03-01;337:109394.

[51] Gnanaprakasam J, Lopez-Banuelos L, Vega L. Anacardic 6-pentadecyl salicylic acid induces apoptosis in breast cancer tumor cells, immunostimulation in the host and decreases blood toxic effects of taxol in an animal model. Toxicol Appl Pharmacol. 2021 2021-01-01;410:115359.

[52] Leandro J, Houten SM. The lysine degradation pathway: Subcellular compartmentalization and enzyme deficiencies. MOL GENET METAB. 2020 2020-09-01;131(1-2):14-22.

[53] Knorr S, Sinn M, Galetskiy D, Williams RM, Wang C, Muller N, et al. Widespread bacterial lysine degradation proceeding via glutarate and L-2-hydroxyglutarate. NAT COMMUN. 2018 2018-11-29;9(1):5071.

[54] Yang CS, Kim JJ, Lee HM, Jin HS, Lee SH, Park JH, et al. The AMPK-PPARGC1A pathway is required for antimicrobial host defense through activation of autophagy. AUTOPHAGY. 2014 2014-05-01;10(5):785-802.

[55] Takano AP, Diniz GP, Barreto-Chaves ML. AMPK signaling pathway is rapidly activated by T3 and regulates the cardiomyocyte growth. MOL CELL ENDOCRINOL. 2013 2013-08-25;376(1-2):43-50.

[56] Castilla-Ortega E, Serrano A, Blanco E, Araos P, Suarez J, Pavon FJ, et al. A place for the hippocampus in the cocaine addiction circuit: Potential roles for adult hippocampal neurogenesis. Neurosci Biobehav Rev. 2016 2016-07-01;66:15-32.

[57] Dan Z, Mao X, Liu Q, Guo M, Zhuang Y, Liu Z, et al. Altered gut microbial profile is associated with abnormal metabolism activity of Autism Spectrum Disorder. Gut Microbes. 2020 2020-09-02;11(5):1246-67.

[58] Jing N, Liu X, Jin M, Yang X, Hu X, Li C, et al. Fubrick tea attenuates high-fat diet induced fat deposition and metabolic disorder by regulating gut microbiota and caffeine metabolism. FOOD FUNCT. 2020 2020-08-01;11(8):6971-86.

[59] Galvez E, Iljazovic A, Amend L, Lesker TR, Renault T, Thiemann S, et al. Distinct Polysaccharide Utilization Determines Interspecies Competition between Intestinal Prevotella spp. CELL HOST MICROBE. 2020 2020-12-09;28(6):83852.

[60] De Filippis F, Pasolli E, Tett A, Tarallo S, Naccarati A, De Angelis M, et al. Distinct Genetic and Functional Traits of Human Intestinal Prevotella copri Strains Are Associated with Different Habitual Diets. CELL HOST MICROBE. 2019 2019-0313;25(3):444-53.

[61] Segata N, Izard J, Waldron L, Gevers D, Miropolsky L, Garrett WS, et al. Metagenomic biomarker discovery and explanation. GENOME BIOL. 2011 2011-06-24;12(6):R60.

\section{Tables}


Table1: Demographic characteristics of monks and control samples in this study.

\begin{tabular}{|c|c|c|c|}
\hline $\begin{array}{l}\text { Variables } \\
\text { median (min-max) }\end{array}$ & Monks $₫ n=24 \rrbracket$ & Controls $\llbracket n=48 \rrbracket$ & $P$-value \\
\hline Age, years & $35.5 \rrbracket 26-55 \rrbracket$ & $26 \rrbracket 24-43 \rrbracket$ & $>0.05$ \\
\hline $\mathrm{BW}, \mathrm{kg}$ & $58 \unrhd 50-78 \rrbracket$ & $50 \rrbracket 44-71 \rrbracket$ & $>0.05$ \\
\hline $\mathrm{BMI}, \mathrm{kg} / \mathrm{m}^{2}$ & $19.90 \otimes 17.13-27.64 \rrbracket$ & $19.63 \rrbracket 16.96-24.22 \rrbracket$ & $>0.05$ \\
\hline The lasting-time of practice & $7.5 \rrbracket 1-27 \rrbracket$ & 0 & / \\
\hline
\end{tabular}

Table2: The detailed information of eight common KEGG categories by bothTax4Fun and PICRUSt2.

\begin{tabular}{|lllllll|}
\hline $\begin{array}{l}\text { KEGG } \\
\text { Pathway } \\
\text { level1 }\end{array}$ & $\begin{array}{l}\text { KEGG } \\
\text { Pathway } \\
\text { level2 }\end{array}$ & KEGG Pathway level3 & Groups & $\begin{array}{l}\text { Log } \\
\text { value }\end{array}$ & $\begin{array}{l}\text { LDA } \\
\text { values }\end{array}$ & P-value \\
\hline Metabolism & $\begin{array}{l}\text { Carbohydrate } \\
\text { metabolism }\end{array}$ & Glycolysis_Gluconeogenesis & Control & 3.9969 & 2.6795 & $<0.001$ \\
\hline Metabolism & $\begin{array}{l}\text { Carbohydrate } \\
\text { metabolism }\end{array}$ & Pentosephosphatepathway & Control & 3.9416 & 2.7843 & $<0.001$ \\
\hline $\begin{array}{l}\text { Environmental } \\
\text { Information } \\
\text { Processing }\end{array}$ & $\begin{array}{l}\text { Membrane } \\
\text { transport }\end{array}$ & ABCtransporters & Control & 4.9684 & 4.0230 & $<0.001$ \\
\hline $\begin{array}{l}\text { Environmental } \\
\text { Information } \\
\text { Processing }\end{array}$ & $\begin{array}{l}\text { Membrane } \\
\text { transport }\end{array}$ & Phosphotransferasesystem_PTS_ & Control & 3.8822 & 3.3047 & $<0.001$ \\
\hline Metabolism & $\begin{array}{l}\text { Carbohydrate } \\
\text { metabolism }\end{array}$ & Citratecycle_TCAcycle_ & Monk & 3.7071 & 2.5261 & $<0.001$ \\
\hline Metabolism & $\begin{array}{l}\text { Glycan } \\
\text { biosynthesis } \\
\text { and } \\
\text { metabolism }\end{array}$ & Otherglycandegradation & Monk & 4.0702 & 3.3150 & $<0.001$ \\
\hline Metabolism & $\begin{array}{l}\text { Glycan } \\
\text { biosynthesis } \\
\text { and } \\
\text { metabolism }\end{array}$ & Lipopolysaccharidebiosynthesis & Monk & 3.9069 & 3.2622 & $<0.001$ \\
\hline Enetabolism & $\begin{array}{l}\text { Energy } \\
\text { metabolism }\end{array}$ & Carbonfixationpathwaysinprokaryotes & Monk & 3.9962 & 2.745197399 & $<0.001$ \\
\hline
\end{tabular}

Table3: Demographic characteristics of monks in the $\mathrm{H}$ and $\mathrm{L}$ group in this study.

\begin{tabular}{|c|c|c|c|}
\hline $\begin{array}{l}\text { Variables } \\
\text { median (min-max) }\end{array}$ & H group $\ n=12 \rrbracket$ & L group $\varangle n=12 \nabla$ & $P$-value \\
\hline Age, years & $37(26-55)$ & 33 (27-54) & 0.443 \\
\hline $\mathrm{BW}, \mathrm{kg}$ & $57.5(50-78)$ & $59(50-65)$ & 0.807 \\
\hline $\mathrm{BMI}, \mathrm{kg} / \mathrm{m} 2$ & $19.90(18.31-27.64)$ & $19.85(17.13-22.49)$ & 0.537 \\
\hline The lasting-time of practice & $12(8-27)$ & $4(1-7)$ & $<0.001$ \\
\hline
\end{tabular}

Table4: The twelve KEGG categories between the two groups by the LEfSe analysis $(\operatorname{LAD}>2.0, p<0.05)$ in Tax4Fun. 


\begin{tabular}{|c|c|c|c|c|c|c|}
\hline $\begin{array}{l}\text { KEGG } \\
\text { Pathway } \\
\text { level1 }\end{array}$ & KEGG Pathway level2 & KEGG Pathway level3 & Groups & $\begin{array}{l}\text { Log } \\
\text { value }\end{array}$ & $\begin{array}{l}\text { LDA } \\
\text { values }\end{array}$ & $P$-value \\
\hline Metabolism & $\begin{array}{l}\text { Carbohydrate } \\
\text { metabolism }\end{array}$ & Glycolysis / Gluconeogenesis & L & 3.9617 & 2.3515 & 0.0496 \\
\hline Metabolism & Amino acid metabolism & $\begin{array}{l}\text { Valine, leucine and isoleucine } \\
\text { degradation }\end{array}$ & $\mathrm{H}$ & 3.5382 & 2.2648 & 0.0282 \\
\hline Metabolism & $\begin{array}{l}\text { Metabolism of other } \\
\text { amino acids }\end{array}$ & $\begin{array}{l}\text { Phosphonate and } \\
\text { phosphinate metabolism }\end{array}$ & $\mathrm{H}$ & 3.1666 & 2.4480 & 0.03767 \\
\hline Metabolism & $\begin{array}{l}\text { Metabolism of other } \\
\text { amino acids }\end{array}$ & D-Alanine metabolism & L & 3.5651 & 2.4300 & 0.00791 \\
\hline Metabolism & $\begin{array}{l}\text { Glycan biosynthesis and } \\
\text { metabolism }\end{array}$ & Peptidoglycan biosynthesis & L & 4.2700 & 3.0967 & 0.0282 \\
\hline Metabolism & Energy metabolism & $\begin{array}{l}\text { Carbon fixation in } \\
\text { photosynthetic organisms }\end{array}$ & L & 3.6891 & 2.5206 & 0.0179 \\
\hline Metabolism & $\begin{array}{l}\text { Metabolism of cofactors } \\
\text { and vitamins }\end{array}$ & $\begin{array}{l}\text { Nicotinate and nicotinamide } \\
\text { metabolism }\end{array}$ & L & 3.9533 & 2.8163 & 0.0130 \\
\hline Metabolism & $\begin{array}{l}\text { Metabolism of cofactors } \\
\text { and vitamins }\end{array}$ & Biotin metabolism & $\mathrm{H}$ & 3.7366 & 2.4594 & 0.0282 \\
\hline Metabolism & $\begin{array}{l}\text { Metabolism of cofactors } \\
\text { and vitamins }\end{array}$ & $\begin{array}{l}\text { Porphyrin and chlorophyll } \\
\text { metabolism }\end{array}$ & $\mathrm{H}$ & 4.2867 & 3.30840 & 0.0433 \\
\hline $\begin{array}{l}\text { Organismal } \\
\text { Systems }\end{array}$ & $\begin{array}{l}\text { Environmental } \\
\text { adaptation }\end{array}$ & Plant-pathogen interaction & $\mathrm{H}$ & 3.4164 & 2.09590 & 0.0209 \\
\hline $\begin{array}{l}\text { Organismal } \\
\text { Systems }\end{array}$ & Nervous system & Dopaminergic synapse & L & 1.0043 & 2.1670 & 0.0243 \\
\hline $\begin{array}{l}\text { Human } \\
\text { Diseases }\end{array}$ & $\begin{array}{l}\text { Infectious disease: } \\
\text { bacterial }\end{array}$ & Pertussis & $\mathrm{H}$ & 3.4150 & 2.7847 & 0.0496 \\
\hline
\end{tabular}

Table5: The twelve KEGG categories between the two groups by the LEfSe analysis (LAD>2.0, $p<0.05)$ in PICRUSt2. 


\begin{tabular}{|c|c|c|c|c|c|c|}
\hline KEGG & KEGG & KEGG & Groups & $\begin{array}{l}\text { Log } \\
\text { value }\end{array}$ & $\begin{array}{l}\text { LDA } \\
\text { values }\end{array}$ & P-value \\
\hline Pathway level 1 & Pathway level2 & Pathway level3 & & & & \\
\hline Metabolism & $\begin{array}{l}\text { Carbohydrate } \\
\text { metabolism }\end{array}$ & $\begin{array}{l}\text { Fructose and mannose } \\
\text { metabolism }\end{array}$ & $\mathrm{H}$ & 3.9069 & 2.7463 & 0.0243 \\
\hline Metabolism & Energy metabolism & Photosynthesis & $\mathrm{L}$ & 3.6048 & 2.2871 & 0.0496 \\
\hline Metabolism & Nucleotide metabolism & Purine metabolism & $\mathrm{L}$ & 4.2003 & 2.5200 & 0.0243 \\
\hline Metabolism & $\begin{array}{l}\text { Amino acid } \\
\text { metabolism }\end{array}$ & $\begin{array}{l}\text { Glycine, serine and threonine } \\
\text { metabolism }\end{array}$ & $\mathrm{H}$ & 3.9769 & 2.1442 & 0.0004 \\
\hline Metabolism & $\begin{array}{l}\text { Amino acid } \\
\text { metabolism }\end{array}$ & $\begin{array}{l}\text { Valine, leucine and } \\
\text { isoleucine degradation }\end{array}$ & $\mathrm{H}$ & 3.2938 & 2.2486 & 0.0209 \\
\hline Metabolism & $\begin{array}{l}\text { Glycan biosynthesis } \\
\text { and metabolism }\end{array}$ & Peptidoglycan biosynthesis & L & 3.9632 & 2.5135 & 0.0282 \\
\hline Metabolism & $\begin{array}{l}\text { Carbohydrate } \\
\text { metabolism }\end{array}$ & $\begin{array}{l}\text { Glyoxylate and dicarboxylate } \\
\text { metabolism }\end{array}$ & $\mathrm{H}$ & 3.8773 & 2.5185 & 0.0377 \\
\hline Metabolism & $\begin{array}{l}\text { Carbohydrate } \\
\text { metabolism }\end{array}$ & Butanoate metabolism & $\mathrm{H}$ & 3.7726 & 2.3330 & 0.0496 \\
\hline Metabolism & Energy metabolism & Sulfur metabolism & L & 3.4790 & 2.1062 & 0.0067 \\
\hline Metabolism & $\begin{array}{l}\text { Global and overview } \\
\text { maps }\end{array}$ & $\begin{array}{l}\text { Microbial metabolism in } \\
\text { diverse environments }\end{array}$ & $\mathrm{H}$ & 4.6444 & 2.7764 & 0.0153 \\
\hline Metabolism & $\begin{array}{l}\text { Global and overview } \\
\text { maps }\end{array}$ & Carbon metabolism & $\mathrm{H}$ & 4.4360 & 2.5092 & 0.03767 \\
\hline $\begin{array}{l}\text { Genetic } \\
\text { Information } \\
\text { Processing }\end{array}$ & Replication and repair & Nucleotide excision repair & L & 3.5929 & 2.0454 & 0.0130 \\
\hline
\end{tabular}

\section{Figures}

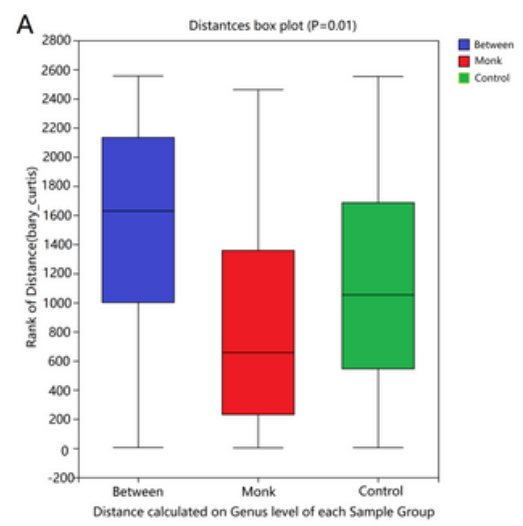

D

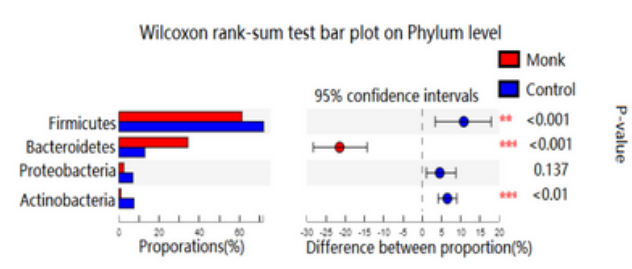

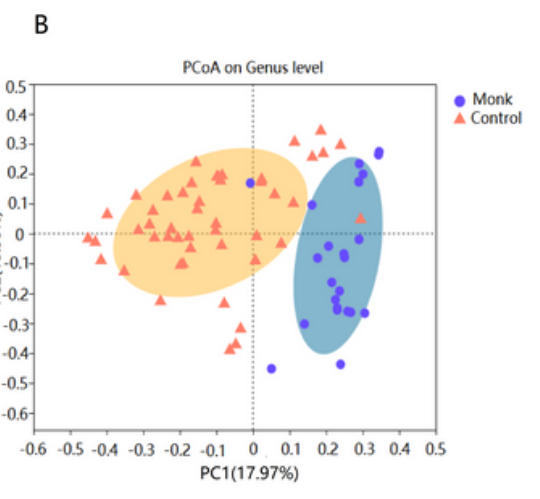

E

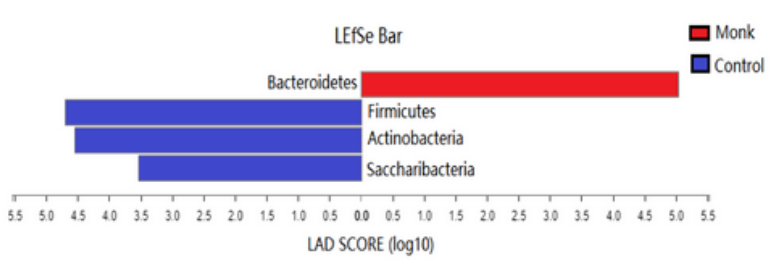

C
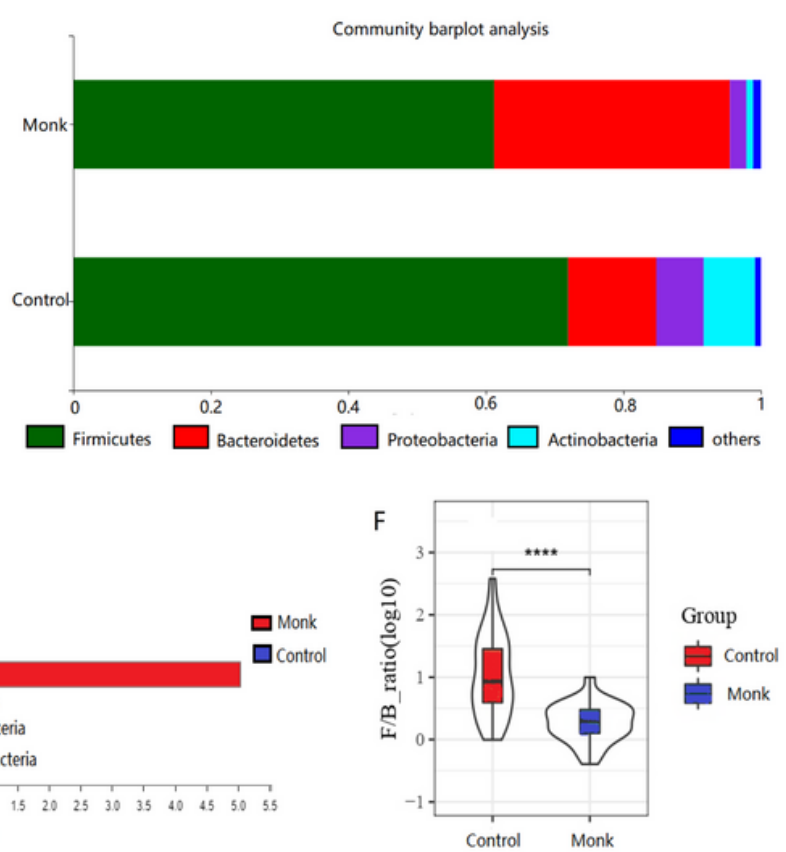
Figure 1

Altered composition of gut microbiota in monks compared with controls at the phylum levels. (A) Beta-diversity analysis showed that the difference between the monks and the controls was significant $(p=0.01)$ at the genus level. (B) PCoA analysis revealed that the microbial composition was clearly different between monks and controls. One dot in the figure represents one sample. (C) Composition of the gut microbiota at the phylum levels. (D) Comparison between the two groups at the phylum levels. ${ }^{\star \star} p<0.01 ;{ }^{* \star} p<0.001$. (E) The LEfSe was used to identify the species that significantly differed between the two groups at the phylum levels (LDA score $>3.0, p<0.05)$. (E) Comparison of Firmicutes/Bacteroidetes (F/B) ratio $(\log 10)$ in monks and controls.
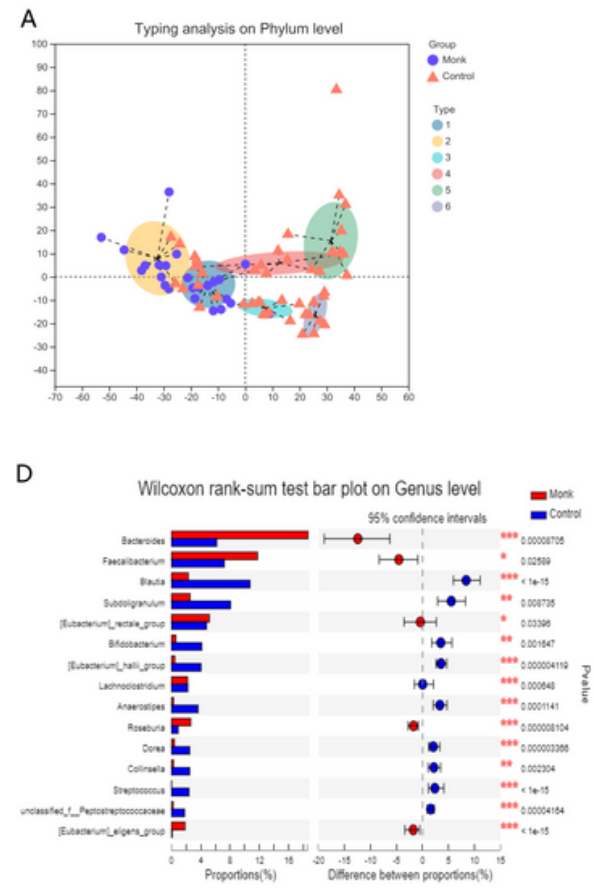
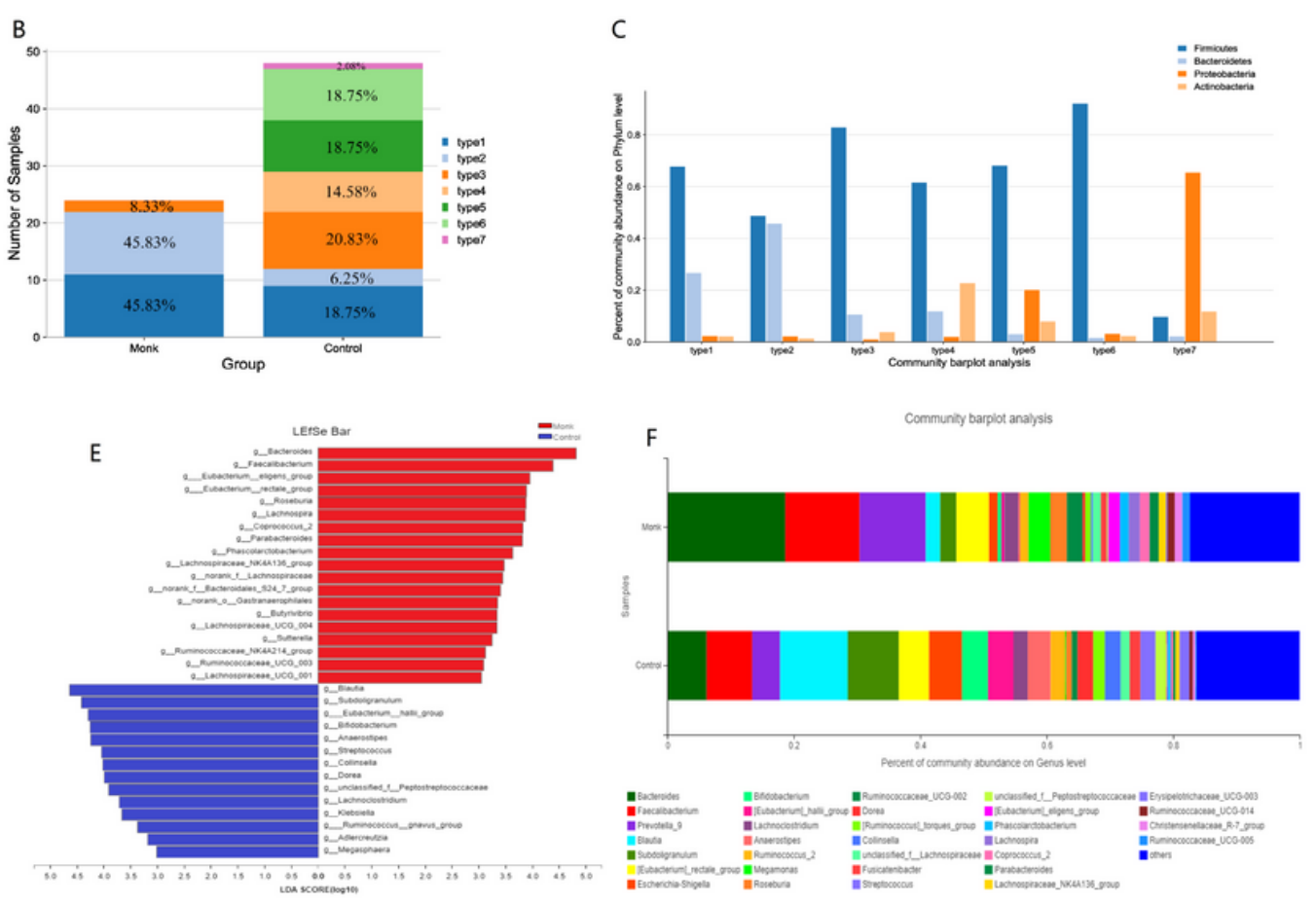

Figure 2

Altered composition of gut microbiota in the two groups at the phylum and genus levels. (A) The distribution of samples in the seven cluster types is presented. The bar-plots show (B) the proportions of cases of different types in the two groups and (C) the relative abundances of the four dominant phyla in the seven types. (D) Comparison between the two groups at the genus levels. ${ }^{\star} p<0.05 ;{ }^{* \star} p<0.01 ;{ }^{* \star} p<0.001$. (E) The LEfSe was used to identify the species that significantly differed between the two groups at the genus levels ( $L D A$ score $>3.0, p<0.05$ ). (F) Composition of the gut microbiota at the genus levels. 

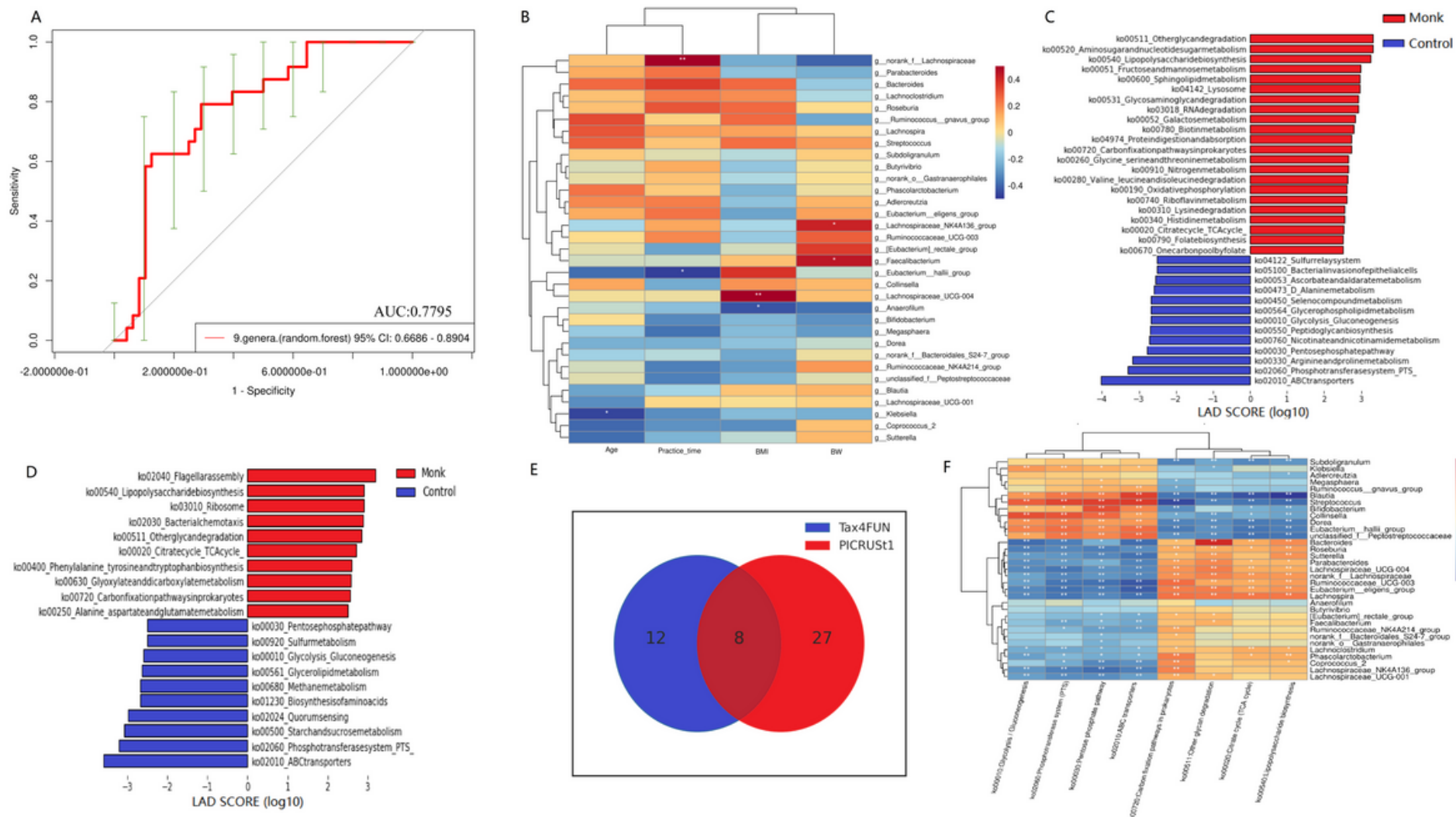

$\mathrm{E}$
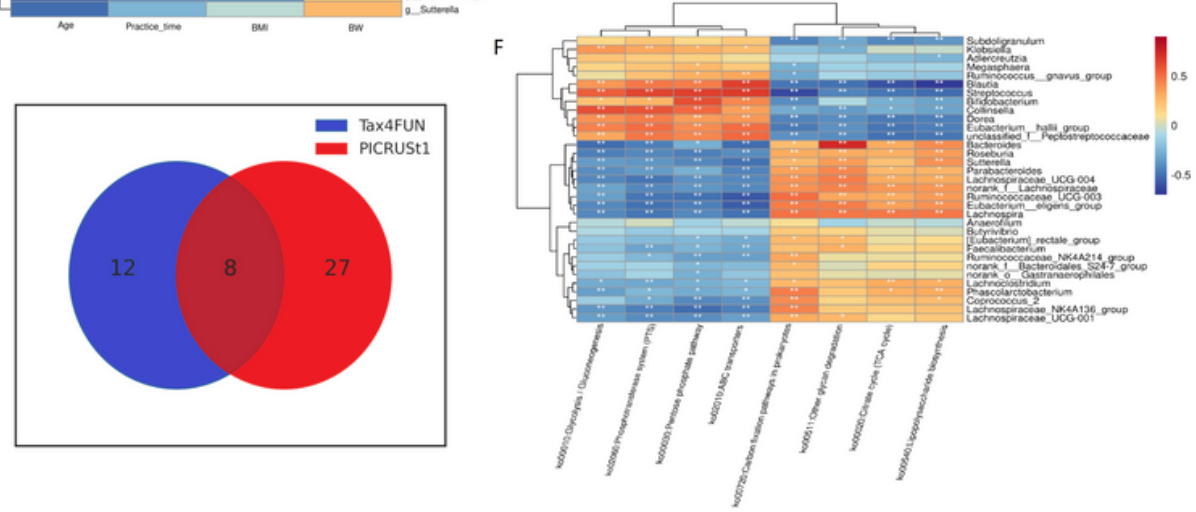

Figure 3

The relationship between gut microbiota and functional analysis prediction. (A) The ROC curve was used to assess the diagnostic accuracy of the top nine genera based on LEfSe results. (B) Spearson correlation analysis was used to determine the relationships between 33 differential genera and monks' general characteristics. Red represents a positive correlation, and green represents a negative correlation. ${ }^{*} p<0.05 ;{ }^{*} p<0.01 ;{ }^{* \star} p<0.001$. The LEfSe analysis ( $\left.\operatorname{LAD}>2.0, p<0.05\right)$ showed that (C) 35 categories of Tax4Fun and (D) 20 categories of PICRUSt2 were significantly differed between the groups. (E) The Venn plot showed the predicted KEGG function counts for the two groups based on Tax4Fun and PICRUSt2. (F) Spearson correlation analysis was used to determine the relationships between 33 differential genera and the eight identified common KEGG categories. 
A

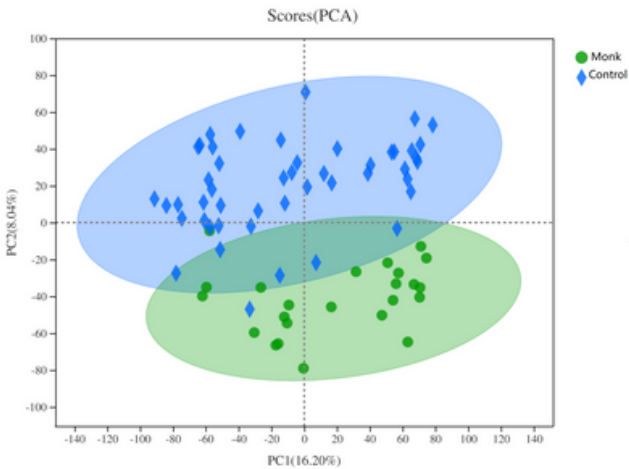

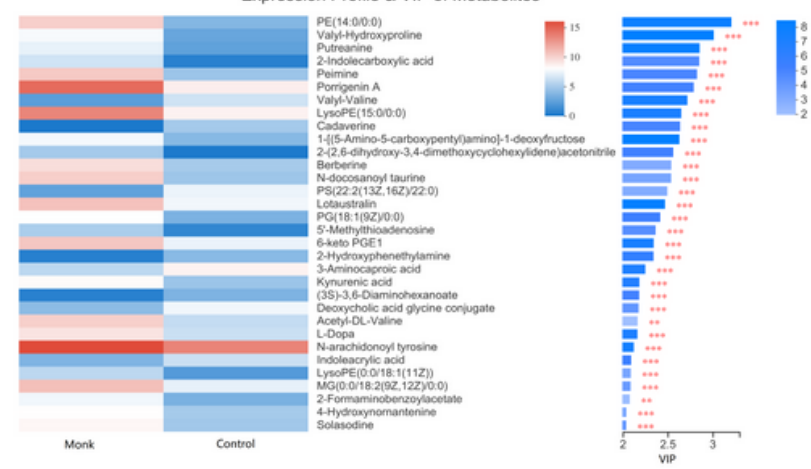

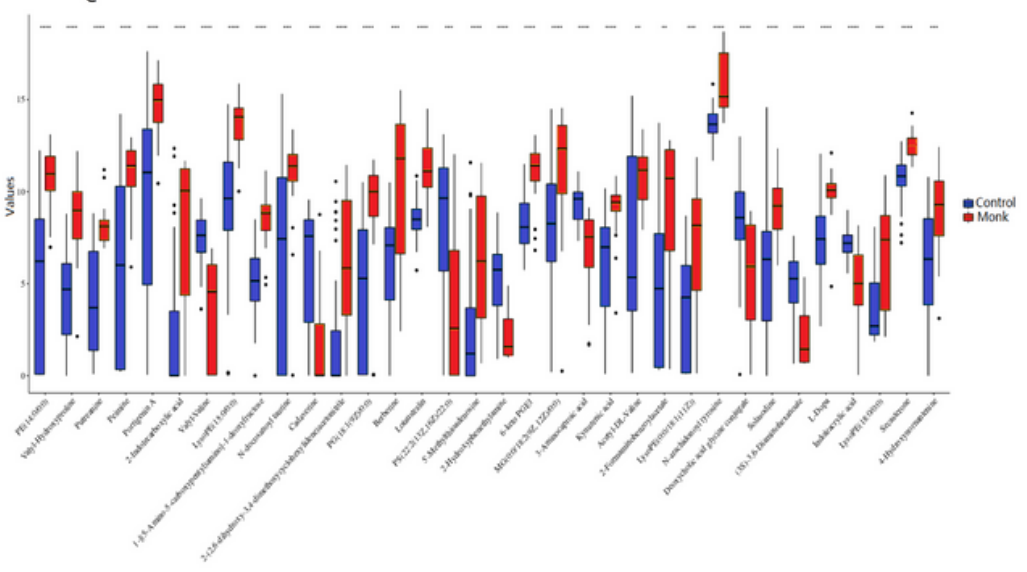

Expression Profile \& VIP of Metabolites

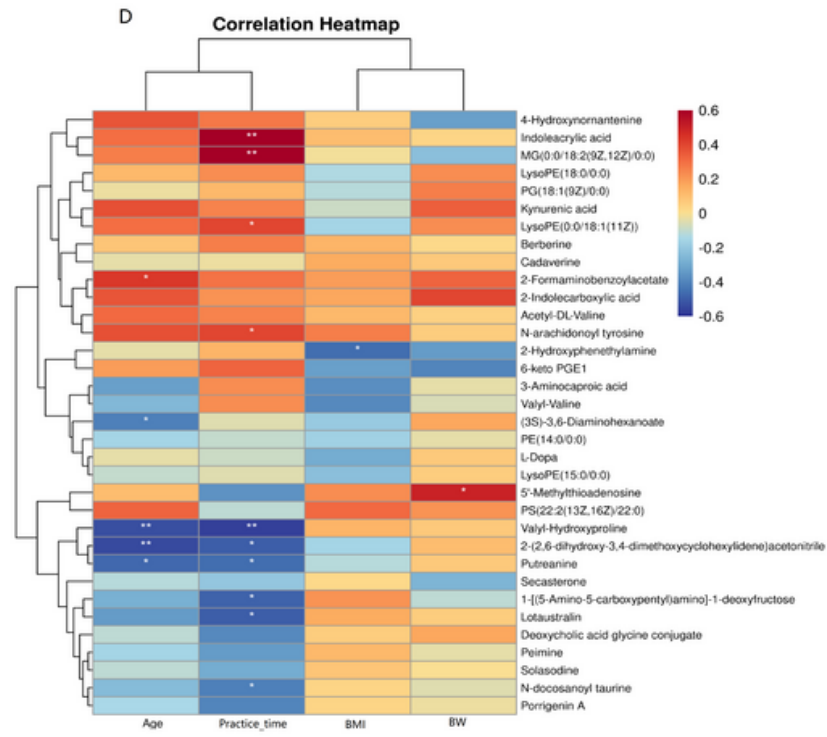

Figure 4

Altered composition of intestinal metabolites in monks compared with controls. (A) PCA model was used to identify the distribution characteristics of metabolites between the groups. (B) VIP analysis ( VIP $>2, p<0.05$ ) was performed to visually display the importance of differential metabolites in the two groups and the trend of expression. (C) The Kruskal test was used to display the relative abundances of all differential metabolites in monks and controls. (D) Spearson correlation analysis was used to determine the relationships between differential gut metabolites and monks' general characteristics. 

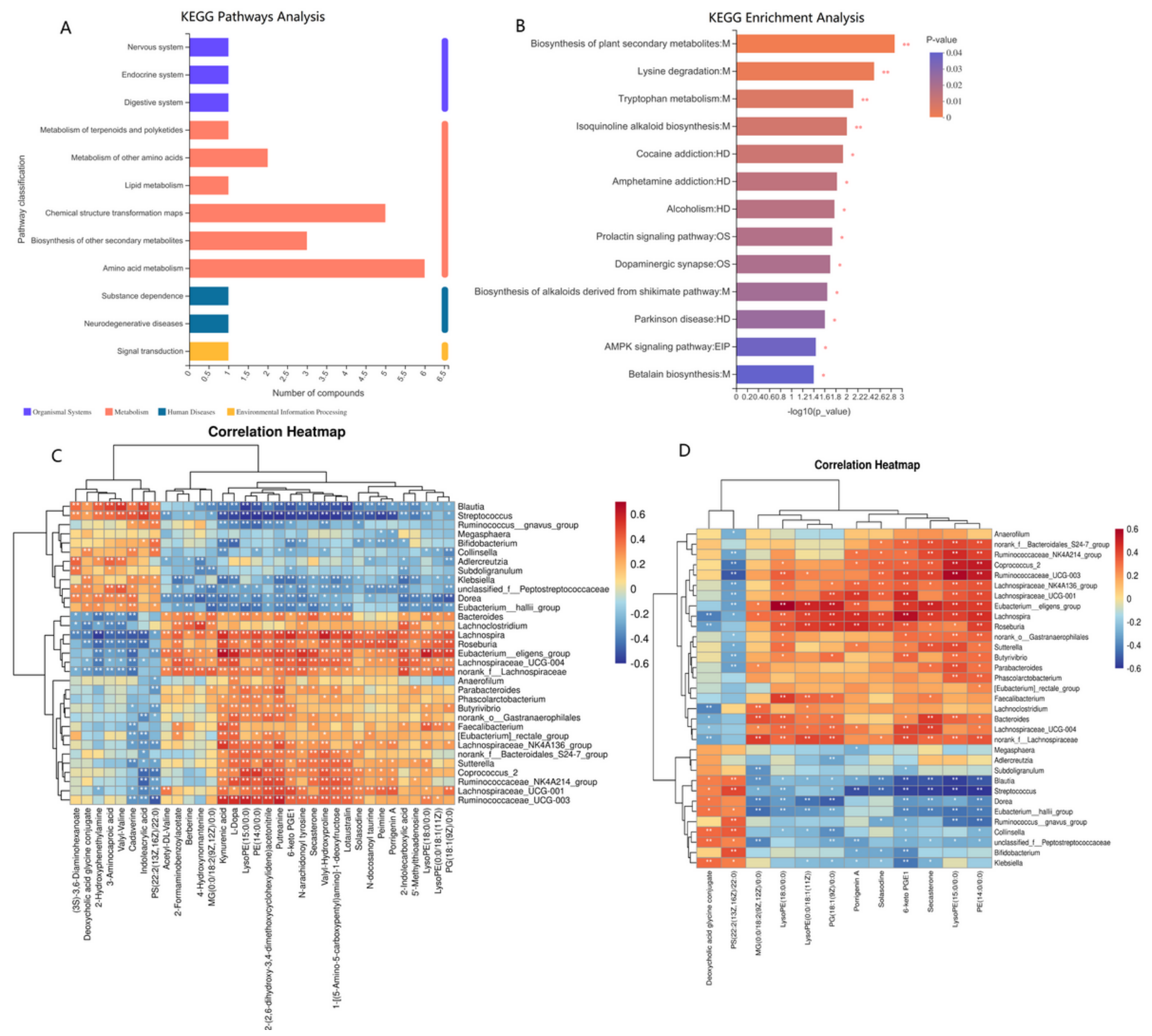

Figure 5

(A) KEGG functional annotation analysis and (B) KEGG pathways enrichment analysis displayed that 23 differential metabolites were involved in the twelve KEGG annotation pathways at level2 and the thirteen KEGG metabolic pathways ( $p<$ 0.05 ). Spearman correlation was used to determine (C) the relationships between 33 differential genera and 34 differential metabolites, and (D) the relationships between 33 differential genera twelve lipids and lipid-like metabolites. 


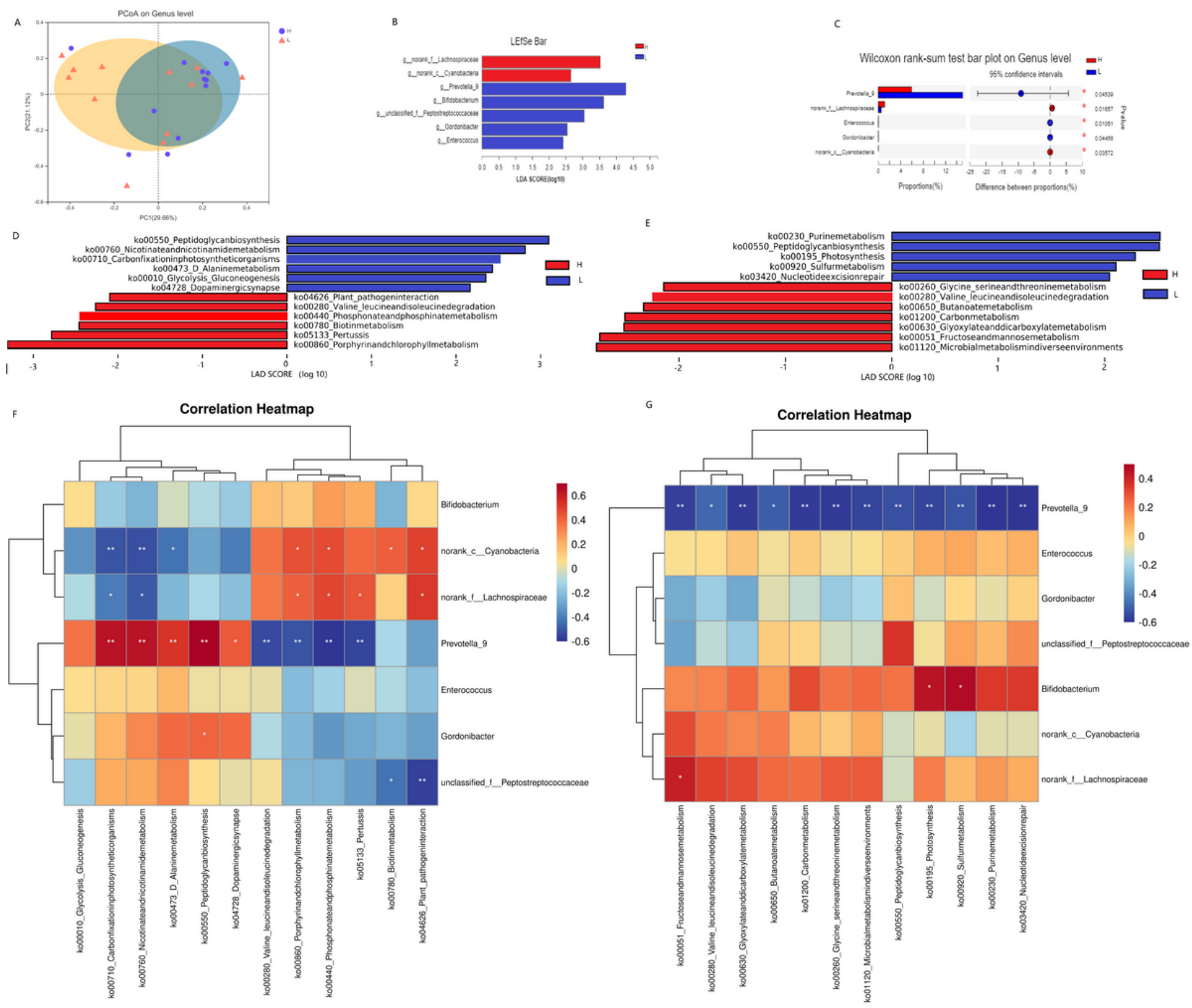

Figure 6

Altered composition of gut microbiota and the Functional analysis prediction in the two groups (A) PCoA analysis revealed that the microbial composition was clearly different between $\mathrm{H}$ group and $\mathrm{L}$ group. (B)The LEfSe was used to identify the species that significantly differed between the two groups at the genus levels ( LDA score $>2.5, p<0.05$ ). (C) Comparison between $\mathrm{H}$ group and $\mathrm{L}$ group at the genus levels. ${ }^{*} p<0.01$; ${ }^{* \star} p<0.001$. The LEfSe analysis $(\operatorname{LAD}>2.0, p<0.05)$ showed that (D) twelve categories of Tax4Fun and (E) twelve categories of PICRUSt2 were significantly differed between the two groups. The Spearman correlation was utilized to analyze the potential connections between the seven differential genera and $(F)$ twelve categories of Tax4Fun and (G) twelve categories of PICRUSt2. 


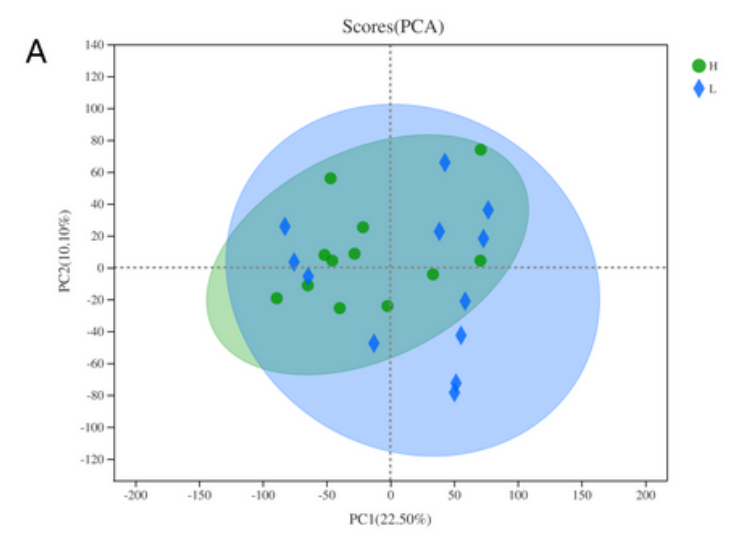

C
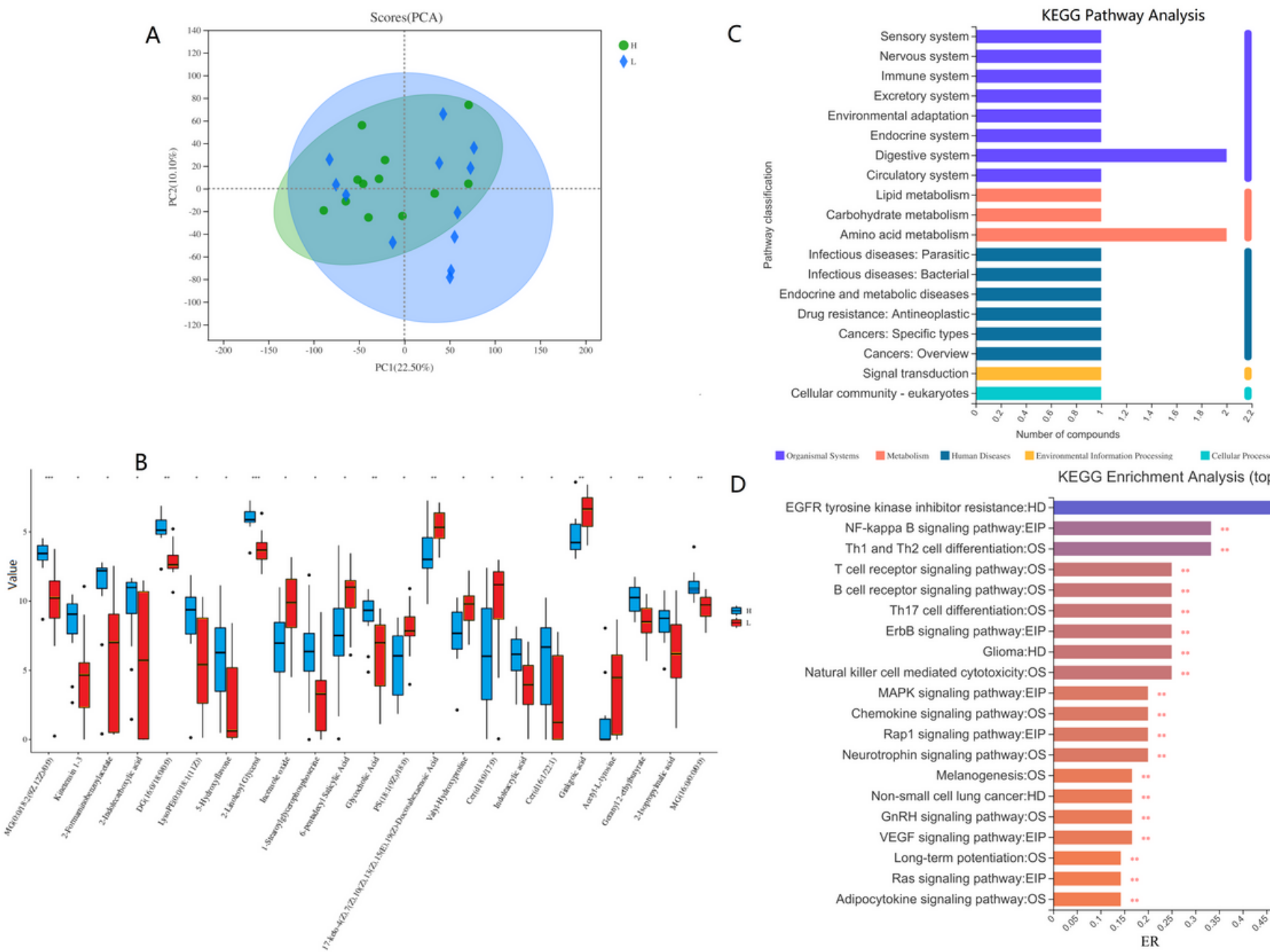

D

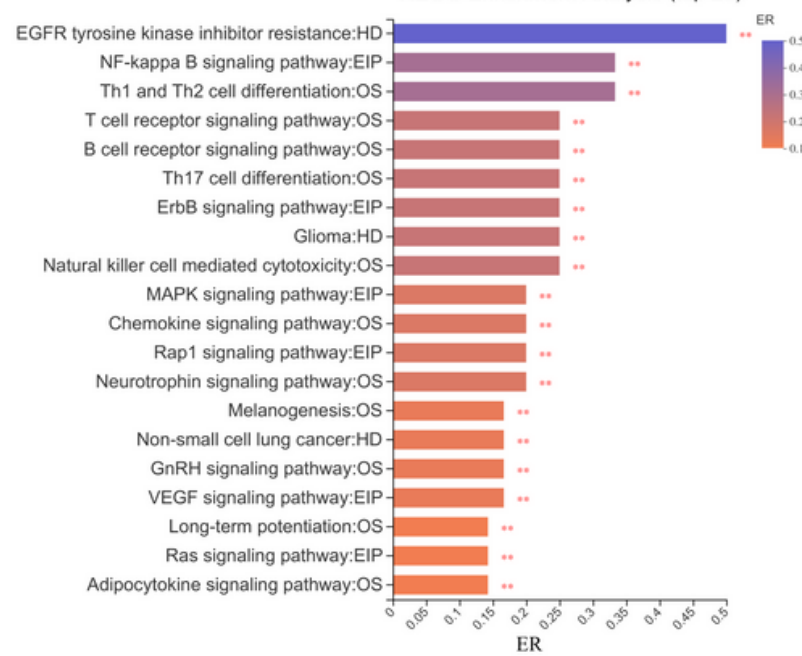

Figure 7

Analysis of metabolites, KEGG functional annotation analysis, and KEGG pathways enrichment analysis in $\mathrm{H}$ group and $\mathrm{L}$ group. (A) PCA model indicated that the $\mathrm{H}$ group and the $\mathrm{L}$ group were different from each other. (B) The Kruskal test displayed the relative abundances of all differential metabolites in the two groups. (C) KEGG functional annotation analysis and (D) KEGG pathways enrichment analysis showed that twelve differential metabolites were involved in five primary KEGG annotation pathways containing 19 secondary pathways and the top $20 \mathrm{KEGG}$ enrichment pathways in ER values $(p<0.05)$.

\section{Supplementary Files}

This is a list of supplementary files associated with this preprint. Click to download.

- TableS1.docx

- Tables2.docx

- Tables3.docx

- Tables4.docx

- TableS5.docx 